\section{To sequence or not to sequence the whole-soil metagenome?}

\author{
Philippe C. Baveye
}

The recent editorial by Timothy Vogel et al. (TerraGenome: a consortium for the sequencing of a whole-soil metagenome. Nature Rev. Microbiol. 7, 252 (2009)) ${ }^{1}$ appears to represent a departure from the previous literature on sequencing soil metagenomes. In this editorial, the usual arguments are presented about the welldocumented lack of understanding of many soil processes that contribute, for example, to environmental and climate change. As other authors have done repeatedly in recent years ${ }^{2,3}$, Vogel et al. emphasize the fact that "Although microorganisms are responsible for key functions in soils, only a small percentage (less than $0.5 \%$ ) have been grown in the laboratory and genome sequences are only available for a select few." However, unlike other articles that start with these same preambles and move on to describe the advantages of metagenomics, Vogel et al. are careful not to present metagenomics explicitly as a way to resolve the problem of unculturable microorganisms. On the contrary, they acknowledge that they "...will need to develop and apply new approaches to cultivate the previously uncultivated and rare members of the soil community to assign functions to the vast number of unknown or hypothetical genes that will undoubtedly be found."

This candid admission suggests a form of circularity in the traditional reasoning behind whole-soil metagenome sequencing projects. Many readers of this editorial are likely to be left with the impression that, when all is said and done and after large financial resources have been spent on developing the appropriate extraction methods and on sequencing the estimated terabases of the metagenome in the Park Grass soil (Rothamsted, United Kingdom), one will be back to square one, faced with the task of having to deal with the $99.5 \%$ of microorganisms that are still unculturable, or at least uncultured, to make sense of the metagenomic data. At that point, as well as a sizeable effort to finally culture these uncultured organisms, additional large pots of money will be needed to carry out indispensable research efforts in other 'meta-omics' approaches (for example, metatranscriptomics, metaproteomics and metametabolomics) $)^{4-6}$ to obtain information that complements the metagenomic data.

In the absence of clear indications from metagenomics researchers that the scenario described above is not going to unfold, the scientific community should perhaps reflect on whether the proposed sequencing of the whole-soil metagenome is a wise step to take at this stage or a good way to spend scarce research money, even though it is technically feasible and regardless of the opportunity that it affords to keep financially struggling sequencing facilities in business. Alternative approaches such as traditional ecological research, complemented by molecular biology techniques ${ }^{7,8}$, may ultimately yield better results, especially if they were to be carried out in the context of current work on the spatial microheterogeneity of soils and emergent behaviours at the macroscopic scale. Recent research $^{9-12}$ indicates that, under various circumstances in soils, bulk (spatially averaged) measurements provide insufficient or even misleading information, a feature that may be shared by the outcomes of metagenome sequencing. From this perspective, spatially targeted metagenomics might be a better step forward than whole-soil metagenomics, provided that one could design methods to sample microorganisms at a fine resolution in soils and then analyse their genetic make-up. Another appealing alternative to wholesoil metagenomics is functionally targeted metagenomics, carried out by substratespecific labelling of microbial DNA using stable isotope probing ${ }^{13}$.

At this juncture, it seems that the field of environmental microbiology would benefit from an in-depth debate in which the various interested parties, including molecular biologists, microbial ecologists and non-microbiologists, present and discuss, in detail, the ultimate, true benefits of whole-soil metagenomics, before proceeding head-first with this time-consuming and potentially onerous operation.

Philippe C. Baveye is at the SIMBIOS Centre, Abertay
University, Kydd Building, 40 Bell Street,
Dundee, DD11HG,
UK.
e-mail: p.baveye@abertay.ac.uk
Vogel, T. M. et al. TerraGenome: a consortium for the sequencing of a soil metagenome. Nature Rev. Microbiol. 7, 252 (2009).

2. Guazzaroni, M. E., Beloqui, A., Golyshin, P. N. \& Ferrer, M. Metagenomics as a new technological tool to gain scientific knowledge. World J. Microbiol. Biotechnol. 25, 945-954 (2009).

3. Rondon, M. R. et al. Cloning the soil metagenome: a strategy for accessing the genetic and functional diversity of uncultured microorganisms. Appl. Environ Microbiol. 66, 2541-2547 (2000).

4. Raes, J. \& Bork, P. Molecular eco-systems biology: towards an understanding of community function. Nature Rev. Microbiol. 6, 693-699 (2008).

5. Turnbaugh, P. J. \& Gordon, J. I. An invitation to the marriage of metagenomics and metabolomics. Cell 134, 708-713 (2008).

6. Wilmes, P. \& Bond, P. L. Metaproteomics: studying functional gene expression in microbial ecosystems. Trends Microbiol. 14, 92-97 (2006).

7. Hohnstock-Ashe, A. M., Plummer, S. M., Yager, R. M., Baveye, P. \& Madsen, E. L. Further biogeochemical characterization of a trichloroethene-contaminated fractured dolomite aquifer: electron source and microbial communities involved in reductive dechlorination. Environ. Sci. Technol. 35, 4449-4456 (2001).

8. Qureshi, S. et al. Effect of microbial activity on trace element release from sewage sludge. Environ. Sci. Technol. 37, 3361-3366 (2003).

9. Jacobson, A. R., Dousset, S., Andreux, F. \& Baveye, P. C. Electron microprobe and synchrotron X-ray fluorescence mapping of the heterogeneous distribution of copper in high-copper vineyard soils. Environ. Sci. Technol. 41, 6343-6349 (2007).

10. Kinyangi, J. et al. Nanoscale biogeocomplexity of the organomineral assemblage in soil: application of STXM microscopy and C 1s-NEXAFS spectroscopy. Soil Sci. Soc. Am. J. 70, 1708-1718 (2006).

11. Kuka, K., Franko, U. \& Ruhlmann, J. Modelling the impact of pore space distribution on carbon turnover Ecol. Modell. 208, 295-306 (2007).

12. Li, L., Peters, C. A. \& Celia, M. A. Applicability of averaged concentrations in determining geochemical reaction rates in heterogeneous porous media. $\mathrm{Am}$. J. Sci. 307, 1146-1166 (2007).

13. Kalyuzhnaya, M. G. et al. High-resolution metagenomics targets specific functional types in complex microbial communities. Nature Biotech. 26, 1029-1034 (2008). 